\title{
Investigación y estudios de posgrado
}

\author{
Ramiro Lafuente López ${ }^{1}$
}

\section{RESUMEN}

Se rea li zan al gu nas con si de ra cio nes acer ca de los pro ble mas que exis ten en cuan to a las re la cio nes en tre la in ves ti ga ción y los es tu dios de pos grado. Se hace én fa sis en al gu nos as pec tos re la cio na dos con la in ves ti ga ción en Bibliotecología y sus posibles vínculos con el posgrado.

\section{ABSTRACT}

Some con si de ra tions are made con cer ning the problems that exist in the re la tion bet ween re search and post-gradua te stu dies. Em pha sis is gi ven to cer tain as pects re la ted to re search done in the area of li brary scien ce and its pos sible con nection with post-gra duate stu dies.

Existe la tendencia a enfocar la investigación como un medio para ge ne rar co no ci mien tos en aque llas áreas que se con si de ran indispensables para la solución de los múltiples problemas de nuestra sociedad, con la idea de investigar para generar conocimientos capaces de in no vary de sa rrollar produc tos y apli ca ciones téc ni cas. Como tras fon do a esta idea sub ya ce la es pe ran za de que los académicos transmuten, mediante algún proceso alquímico,elconocimientocientífico-técnicoendesarrolloeconómico.

Este en fo que por lo ge ne ral con du ce a pon de rar la in ves ti ga ción tec no ló gi ca en de tri men to de la aca démica. Es de cir, sefor ta le ce la in ves ti ga ción apli ca da a la so lu ción de proble mas es pe cíficos y se dedican pocos espacios a la investigacióndestinadaa generar conocimientos para fundamentar el desarrollo de nue vos conocimientos que ayuden a comprender y explicar la realidad. Existen plan tea mien tos que con si de ran am bos as pec tos de la inves ti ga ción como ac ti vi da des ex clu yen tes, como si la solu ción a los problemas que plantea la investigación en las universidades estuviese en responder a dos preguntas de diversa índole:

1. ¿Se quiere más tecnología? Entonces hagamos girar los programas de investigación en torno a especialidades técnicas, la práctica y su aplicación.

2. ¿Se de sean co nocimien tos úti les para com pren der la rea li dad? Pues orien te mos los pro gra mas de in ves ti ga ción en tor no a proble mas y bus que mos lain ter dis ci plinarie daden lain ves tigación, para lograr una investigación encausada hacia el análisis de la realidad y la generación de conoci mien tos para com pren der lay explicarla, así como conocimientos que nos sirvan para fundamentar el desarrollo de nuevos conocimientos.
Sin em bar go, con si de ra mos que la pre sen cia de la in ves ti ga ción tecnológica y la puramente académica forman parte de la vida cotidiana del quehacer universitario, son elementos indispensables y complementarios. No deben alentarse aquellos planteamientos que involucran una disyuntiva entre la investigación tec noló gi ca y la aca dé mi ca por que en ton ces nos co loca re mos en la po si ción de per der nos el de ba te de fon do acer ca de los problemas que afectan a la investigación bibliotecológica.

Enelámbi to de la Biblio te cología, en don de tra di cional mente se ha prevalencido la investigación orientada hacia la creación de téc ni cas apli ca bles a la so lu ción de los proble mas que pre sen ta la selección y organización documental, resulta no sólo indispensable sino salu dable fo men tar lain ves ti ga ción pu ra men te aca démica. Estoúl ti mocon obje to de lo grar co no ci mien tos que sirvan parasus ten tar una in ves ti ga ción sis te máti ca acerca de las for mas en cómo se presenta la circulación documental en una comunidad, para que la in ves ti ga ción so bre tec no lo gía apli ca da en la organización de documentos y análisis de sus contenidos, cuente con los suficien tes fun da men tos para ofre cer res pues tas ade cuadas a las necesidades propias de la sociedad mexicana.

Lain ves ti gación biblio tecoló gi ca noescapa alosfenómenos que afectan a la investigación en general, sobre todo por lo que respecta a cómo generar conocimientos a partir de la realidad que plantea la sociedad mexicana, de tal forma que la investigación con ver ti daen conoci mien to pue da ser di fun di day asi mi la da por la sociedad.Involucra, también, reconocerque es in dis pensable la pre sen cia de la Uni ver si dad Pú bli ca como ins ti tu ción preocupada y des ti na da a ge ne rar y di fun dir co noci mien tos que in volucren no sólo aquellos as pectos vincula dos ain te reses es pecíficos o círculos sociales determinados, sino principalmente el interés de la sociedad en su conjunto. 
Esto úl ti mo re sul ta re le van te en el cam po de la in ves ti ga ción bibliotecológica, porque involucra aspectos vinculados con la creación de mecanismos (bibliotecas, centros de información y docu men ta ción) orien ta dos a faci li tar la difu sión pública ein discri mi na da de la in for ma ción. Esto no quie re de cir que no exis tan universidades o instituciones públicas o privadas encausadas a investigaciones es pecíficas; es in dis pen sabley salu da ble para la so cie dad su exis ten cia. Pero de be mos evi tar con ce bir es tas cuestiones como elementos que se contraponen. La Universidad como instancia de servi cio pú bli co es más ne ce si dad para el desa rro llo del cuer po so cial en su con jun to, que sir ve como ca ta lizador de problemas que atañen al conglomerado social y al mejoramiento de sus condiciones de vida.

La Uni versi dadPúblicare pre sen ta un en cla ve aca dé mi co den tro de la sociedad mexicana,producto del éxito del liberalismo mexicano, en cuanto a su insistencia de sustentar la educación pública en el uso del método científico y la libertad de cátedra. Su vir tud, y la fuen te de sus pro ble mas, ra di ca en ser una ins ti tu ción que al tratar de llevar a sus últimas consecuencias la libertad de enseñanza-aprendizaje proclamada por el liberalismo, con la úni ca li mi tan te del uso de mé to dos ra ciona les y cien tífi cos como criterio para normar sus actividades, se encierra en graves contra dic ciones, por que den tro de su or ga ni za ción tra ta de con ci liar el igualitarismo producto de la libertad de cátedra, con la jerarquización de riva da de una tra dicional or ganización delquehacer científico y de las nuevas tendencias de la planificación de la ciencia y la educación superior.

Además, des de un pun to de vis ta bi blio te co ló gi co, la li ber tad en el proceso de enseñanza-aprendizaje involucra necesariamente la disponibilidad de acervosdocumentales por medio de bibliotecas abiertas a todo público, para garantizar el ejercicio de la proclamada libertad de enseñanza y de igualdad de oportunidades en el ac ce so a la edu ca ción. No obs tan te, la prác ti ca co ti dia na con sus múltiples dificultades y limitaciones institucionales derivadas de las características que ha ido adquiriendo la administración universitaria, imponen pragmáticamente las orien ta cio nes a la in ves ti ga ción, a la do cen cia y a las po líti cas de formación de acervos documentales.

La estructura actual de las universidades públicas mexicanas basasufuncionamien toenun modeloadminis trativocons ti tuido por una bu ro cra cia en la cús pi de, o cer ca de ella, cuya fun ción es en fren tar a las múl ti ples ins tan cias del go bier no, pre pa rar pla nes y pro gra mas y ade cuar los a la Con ta bi li dad Públi ca para con vencer a los res pon sables del go bier no de que el di ne ro pú bli co que se emplea en la universidad, de alguna manera y por procedimientos misteriosos, se convertirá en algún momento en diversos beneficios sociales y económicos. En sentido estricto, este modelo de organización administrativa tiene mayor significado y tras cen den cia para el de sa rrollo de la in ves ti ga ción y do cen cia, toda vez que no sólo influye en la dinámica y organización del trabajo de investigación, sino, in clu si ve, en la de ter mi na ción de su na tu ra le za y ob je ti vos. Por que si bien la dis ponibili dad y/ocali dad de los re cur sos no cues tio na en prin ci pio las orien ta cio nes metodológicas y/o ideológicas de la investigación-docencia, de algunamanera pue den lle gar a con ver tir se en una li mi tan te para el desarrollo de las mismas.

Como en al gu nas oca sio nes los re cur sos para la in ves ti ga ción se tienen que recabar en distintas dependencias gubernamentales (cada una con sus propios criterios y políticas) se requiere adecuar los proyec tos de in ves ti ga ción a dis tin tos pará me tros. Además, la naturaleza de los mecanismos de control administrativo no siem pre pre veen toda la gama po si ble de las di fe ren tes ne ce sidades de la investigación en distintos campos delconocimiento, lo cual origina la inversión de una buena cantidad de tiempo en ajus tar las me tas y fi nes de la in ves ti ga ción a los re que ri mien tos planteados para el otorgamiento de recursos.

Este tipo de problemas relacionados con prácticas administrativas co ti dia nas afec tan el de sem pe ño de la in ves ti ga ción al fa ci litar la, dis tor sio nar la, de ter mi nar la u obli gar su ade cua ción ante la presencia de recursos inesperados o concedidos a destiempo.

La so lu ción a es tos asun tos no ra di ca ex clu si va men te en el análisis casuístico de los mismos, porque las dificultades no derivan exclusivamente de fallas en la actuación administrativa, sino también de concepciones de control administrativo, que ya no re sul tan ade cua das para en fren tar los re que ri mien tos de la so ciedad ac tual. Es im por tan te com pren der que este pro ce so no afec ta úni ca men te a la ad mi nis tra ción uni ver si taria. Los ad minis tra do res universitarios es posible que tengan más problemas en común con los funcionarios de las grandes empresas o con los administradores del gobierno que con los académicos.

El mo de lo de ad mi nis tra ción uni ver si ta ria for ma par te de la evolu ción his tórica de las uni versi da des occiden tales. Du ran te al gún tiem po rin dió bue nos fru tos en la me di da en que una uni ver si dad pudo contar con un eficiente grupo buscador de fondos para la ins ti tu ción. Su trans for ma ción trajo apa re ja do, como par te de su mismo éxito, al modelo político.

La uni ver si dad lle ga a in cluir lo todo. Los már ge nes en tre la univer si dad y la so cie dad se des di bu jan. Los in te re ses de la vida política se repiten en el microcosmos universitario, que no está preparado para ellos porque según su propia teoría no deberían suceder. La politización del modelo administrativo, condujo al Go bier no a in sis tir en un ma yor con trol de los pre su pues tos univer si ta rios y a un ma yor cre ci mien to del sec tor pú bli co de la educación superior en detrimento del universitario y choques entre los es tu dian tes po li ti za dos y las au to ri da des que tra tan de ex pandir la eficiencia del modelo administrativo.

Un paso ine vi table del mo de lo de ad mi nis tra ción uni ver si ta ria se en cuen tra en di rec ción de la des cen tra li za ción de la toma de decisiones y la creación de un orden reglamentario, que permita a las de pen den cias uni ver si ta rias una ma yor au to no mía y al in vesti ga dor una ade cua da par ti ci pa ción en la toma de de ci sio nes, así como la correspondiente responsabilidad por su actuación. 
En el caso de los es tu dios de pos gra do, la par ti ci pa ción del in vesti ga dor en la toma de de ci sio nes y una cla ra de fi ni ción de los objetivos a perseguir son particularmente clave para enfrentar sus problemas. En el caso de la bibliotecología, el posgrado se ha con ver ti do en una pro lon ga ción de la li cen cia tu ra al tra tar de solucionar los problemas de la especialización y la actualización profesional, al no existir una clara vinculación entre docencia e investigación.

Noexis ten en la ac tuali dad me canis mos coti dia nos que per mitan a los investigadores, cuyo tra ba jo se re la cio na con el ám bi to bibliotecológico, el participar sin mayores complejidades en el pos gra do, no sólo como do cen tes sino in clu so como par te de su formación académica, cuando así lo consideren conveniente para el cumplimiento de sus necesidades y objetivos académi$\cos$.

La orien ta ción del pos gra do en Biblio te co lo gía, en ca mi na doexclusivamente al cum pli mien to de una cu rrícu la y sin po si bi li dades de incluir nexos con los trabajos de investigación, provoca que los tutores académicos del posgrado, que juegan un papel fun da men tal en cuan to a la orien ta ción y for ma ción de los alumnos, se preocupen prioritariamente por hacer que el alumno cubra las materias indispensablespara cumplimentar los objetivos del plan de es tu dios y de jan de lado cual quier otra con si de ra ción, con lo cual el alumno necesariamente se ve condicionado a seguir un plan de estudios rígi do y ex clu yen te de los progra mas de investigación en curso.

Las re la cio nes en tre in ves ti ga ción y es tu dios de pos gra do es una cues tión que se en cuen tra plan tea da en los re gla men tos. Sin embar go, una apre cia ción li ge ra de la rea li dad mues tra que esta rela ción no ha fun cio na do. Y no es una cues tión que po da mos de jar de lado.

La investigaciónpierde algo de su agudeza cuan do se ais la y no exis te la pre sión de co mu ni car co no ci mien tos a las nue vas ge nera cio nes o ha cia otros cam pos dis tin tos de la es pe cia li dad que se maneja.

El posgrado ve reducida su riqueza de posibilidades al circunscribirse a una ac ti vidad do cen te es ca sa men te vin cu la da a los proce sos y re sul ta dos de la in ves ti ga ción en curso. En este sen ti do es que re sul ta salu dable la in te rac ción con los es tu dios de pos gra do. Pero exis ten múl ti ples aris tas del pro ble ma que es ne ce sa rio enfrentar, uno de ellos sería la revalorización de los criterios de evaluación de la investigación, punto particularmente neurálgico para la relación investigación-estudios de posgrado, porque, por su pro pia na tu ra le za, el pos gra do re quie re de ar ti cu lary fundamentar conocimientos en un medio ambiente dentro del cual no exis ten en for ma sis te má ti ca, lo cual re quie re de una bue na inver sión de tiem po, no con tem pla da den tro de los cri te rios ac tuales acerca de la investigación.

Establecer vínculos entre investigación y estudios de posgrado con lle va la ne ce si dad de sis te ma ti zar los re sul ta dos de la in ves tiga ción, para fa ci li tar su apro pia ción y di fu sión, lo cual nos conduce a un indispensable replanteamiento de las actividades de in ves ti ga ción y a in cluir la ela bo ra ción de sín te sis críti cas-au tomatizadas por su nivel académico- como una actividad valiosa para lain ves ti ga ción, por que su obje ti voes fun da mental men te la in ves ti ga ción y la do cen cia. Es de cir, no res pon de a fi nes uti li tarios sino que bus ca la ex pan sión de los co no ci mien tos u no se reduce a proble mases pecífi cos ni sus obje ti voses tán orien ta dos a cor to pla zo o a in ten tos para me jo rar un pro ce so o ser vi cio, sino hacia la elaboración de teorías, esquemas, explicaciones y funda men ta ción del co no ci mien to in dis pen sables parael sano de sarrollo de la docencia y la investigación; sobre todo en áreas del conocimien to comoelbiblio te cológi cocuyos supues tos básicos es tán sien do ob je to de una pro fun da re vi sión en en cla ves aca démicos de distintas partes del mundo.

Un pro ble ma fun da men tal que debe es tar pre sen te en este tipo de cuestiones son los requerimientos de recursos humanos de alto nivelacadémico que con lle va cual quier es fuer zo por me jo rarel pos gra do. En una uni ver si dad como la UNAM no pue de afir marse ca te gó ri ca men te que se dis po ne so bra da men te de es tos re cursos. Ade más, en el caso es pe cífi co de la Bi blio te co lo gía, dado el tradicional én fa sis que los egre sa dos de la li cen cia tu ra ha cen en el ejercicio profesional, los recursos humanos interesados en la in ves tigación y profesional menteca paci ta dos parala mis mason es ca sos. Por ello, es in dis pen sable, tam bién, aten der es tos as pectos sin caer en la ten ta ción de la rá pi da im pro vi sa ción o de so brecargar con actividades docentes a los cuadros de investigación. Ambas posibilidades conllevan graves riesgos que, indudablemente, afectarán la calidad académica a largo plazo, generando problemas de tipo recurrente.

Para solucionar los problemas del posgrado no sólo se requiere de más investigadores, también redefinir y fortalecer la formación de recursos académicos por el único medio probado para hacerlo: tomar personal con el grado y la formación académica in dis pen sable y es table cerunare la ción de en señan za-a pren dizaje, tutelada por aquellos académicos que han probado saber investigar.

Cualquier modificación de la currícula del posgrado, sin contar con los recursos humanos con capacidad académica para analizar la realidad, problematizarla y generar soluciones aprovechan do los co no ci mien tos acu mu la dos, será con ti nuar en la línea de to mar al pos gra do como una prolon ga ción de la li cen cia tura.

El pos gra do debe con si de rar se como un ins tru men to para la formación de recursos humanos para el quehacer académico y no únicamente como la solución a problemas de especialización. Debe ofre cer al alum no la po si bi li dad de es co ger en tre múl ti ples en fo ques para su for ma ción. Debe pro mo ver se la ex plora ción de aquellos caminos que reduzcan el aislamiento de los posgrados en tre sí. Tam bién se debe evi tar que pre va lez ca el en fo que en la formación de tecnólogos, no porque no los necesite la sociedad mexicana, sino porque también existe la urgencia de recursos humanos con formación académica. 
Por otra par te, es in ne ga ble que la di fu sión de los re sul ta dos de la investigación como parte de la actividad docente del posgrado, deja mu cho que de sear. Esto no de ri va pro pia men te de la or ga nización institucional de la investigación y del posgrado, sino de en con trar los me dios ins ti tu cionalesidóneos paralo grardifun dir el co no ci mien to, ya no sólo en tre los alum nos del pos gra do sino, inclusive, entre los mis mos in ves ti ga do res, lo cual no es de fá cil so lu ción pues mien tras la in ves ti ga ción siem pre ac túa so bre aspectos parciales del conocimiento (presuponiendo que el destinatario de la misma conoce todos los aspectos y vinculaciones del asun to de que se tra ta), la do cen cia re quie re de sín te sis de cono ci mien tos bien fun da men ta dos. En este as pec to ten drá que revalorarse la función de la investigación bibliotecológica en cuan to a sus po si bles re la cio nes con los es tu dios de pos gra do, sobre todo en aque llos as pec tos que in volu cran la sis te ma ti za ción y di fu sión de cono ci mien tos ne ce sa rios para un ade cua do de sarrollo del posgrado en bibliotecología.

En este sen ti do per sis te un va cío. Es tas cues tio nes no son pro pias de la uni ver si dad pú bli ca, sino re la ti vas a la es truc tu ra de la co muni ca ción cien tífi ca y es mo ti vo de preo cu pa ción para la bús que da de solu cio nes. Pero de al gu na ma ne ra las po sibles res pues tas que se encuentran a la difusión del conocimiento generado por la inves ti ga ción, para que pue da ser apro ve cha do por la do cen cia y por los mis mos in ves ti ga do res, no es una cues tión que pue da re sol verse de ma ne ra sim plis ta ni a cor to pla zo, ra zón de más para abor darlo de forma consistente y lo más pronto posible.

Todos estos cuestionamientos tienen la intención de destacar la im por tan cia de es ta ble cer ma ti ces en cuan to a la ad mi nis tra ción de la in ves ti ga ción y se ña lar que do tar de ca pa ci dad de de ci sión a las di fe ren tes de pen den cias de in ves ti ga ción no des tru ye el concep to de la uni dad de la Uni ver si dad cuan do, como prin ci pio básico, se reconoce que tanto la autoridad como la capacidad para la toma de de ci siones debe fun da men tar se en la ca li dad aca dé mica de quien la ejerce.

Los problemas políticos y económicos del país hacen acto de pre sen cia en la vida uni ver si ta ria. Ten dre mos que acos tum brarnos a con vi vir con ellos por que es tos pro ble mas no se re du cen a la sim ple y có mo da mi li tan cia par ti dis ta. En el caso de la in ves tiga ción bi blio te co ló gi ca se tra ta de algo más com ple jo: cómo dar res pues ta, den tro de los es tre chos lími tes de las posi bi li da des de ac ción uni ver si ta ria, a aque llos que pre ten den con ver tir a la Univer si dad en ins tru men to de quie nes han acu mu la do y aca pa ra do todo lo re la cio na do a los recur sos in for ma ti vos y docu men tales; al mis mo tiem po se de ben bus car so lu cio nes ade cua das para que la uni ver si dad pú bli ca abra es pa cios para que aque llos que nada tienen puedan aprovechar los conocimientos y la información a través de los servicios públicos bibliotecarios.

Tendrá que hacerse lo necesario para que la Universidad siga com pro me ti da con el in te rés públi co de la so cie dad me xi ca na en su conjunto. Es lo único que la puede mantener vinculada a la realidad. Desvincular las actividades universitarias del análisis de la rea li dad pue de re sul tar có mo do a cor to pla zo, pero con lle va a largo plazo la presencia cada vez más acentuada de la indiferencia.

Una universidad vinculada a la realidad mexicana puede crear conoci mien tos y la so cie dad apro ve char los con for me a los re cursos que tenga a su disposición. Una universidad indiferente y des li ga da de la rea li dad na cio nal, no hará otra cosa que acu mu lar datos que contribuyan a aumentar la frustración social y la dependencia del país.

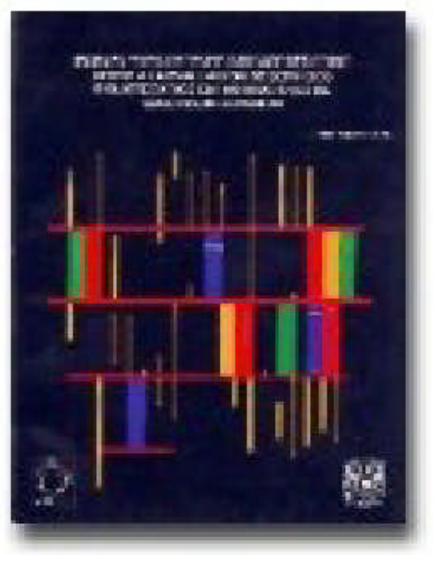

Manual par obtener indicadores como apo yo a la eva lua ción de servicios bibliotecarios en instituciones de educación superior

Pagaza, Rafael. 84 p. 